\title{
Facet structure of GaAs nanowires grown by molecular beam epitaxy
}

\author{
S. O. Mariager, C. B. Sørensen, M. Aagesen, J. Nygård, and R. Feidenhans'|a) \\ Niels Bohr Institute, University of Copenhagen, 2100 Copenhagen, Denmark \\ P. R. Willmott \\ Swiss Light Source, Paul Scherrer Institute, 5232 Villingen, Switzerland
}

(Received 28 May 2007; accepted 17 July 2007; published online 22 August 2007)

\begin{abstract}
The shape and facets of GaAs nanowires grown by molecular beam epitaxy have been identified by the use of the diffuse scattering around the Bragg reflection from the parts of the nanowires having different crystal structures. The zinc blende parts of the nanowires are shown to have $\{111\}$ facets in the form of truncated octahedrons, whereas the facets of the wurtzite structure are $\{10 \overline{1} 0\}$. A minor part of the nanowires have a new structure consisting of a superlattice of regular and twinned structures only six bilayer thick. (C) 2007 American Institute of Physics. [DOI: 10.1063/1.2769401]
\end{abstract}

Freestanding epitaxially grown semiconductor nanowires have attracted large interest in recent years and show promising properties in electronic, photonic, and biological applications. Nanowires have been fabricated by various techniques and special focus has been on the "bottom up" approach where a metal catalyst particle is used to initiate growth. ${ }^{1-3}$ The size of the catalyst particle defines the diameter of the nanowire and a high level of growth control has been achieved ${ }^{4,5}$ though many questions remain to be settled. For instance, it has recently been debated whether or not the catalyst liquify during growth, ${ }^{6,7}$ and simple structural questions such as the orientation of the facets have until recently remained unanswered. In this letter we address the latter question by the use of glancing angle x-ray diffraction. Previously, the characterization techniques have mainly been limited to scanning electron microscopes (SEMs) and transmission electron microscopy (TEM) with a few applications of scanning tunneling microscopy ${ }^{8}$ and $\mathrm{x}$-ray powder diffraction. ${ }^{9}$ For GaAs nanowires, TEM diffraction patterns have been used to identify the crystal structure of the nanowires, which have been found to contain a large amount of twin faults perpendicular to the preferred $[111] B$ growth direction. ${ }^{4,7}$ SEM images have been used to identify the macrofacets from images of the nanowire cross section. The facets depend on the growth direction, ${ }^{4}$ diameter, and As/Ga flux ratio ${ }^{5}$ but for most cases the cross section is hexagonal with $\{11 \overline{2}\}$ surfaces for GaAs nanowires grown in the [111]B direction. Recently, Johansson et al., ${ }^{10}$ however, used TEM images to identify a microfaceted structure of GaP nanowires. In this letter, we report on the use of x-ray diffraction on GaAs nanowires to determine the crystal structure and facets of GaAs nanowires which have not been previously observed for a regular microfaceted structure.

The GaAs nanowires were fabricated in a Varian GEN II molecular beam epitaxy (MBE) machine with effusion cells for $\mathrm{Ga}$ and As. We have introduced a Au effusion cell directly into the growth chamber which allows us to deposit the $\mathrm{Au}$ on an oxide-free clean surface just prior to growth. The GaAs nanowires were grown on a (111)B GaAs surface. After transferring the substrate to the growth chamber it was heated to $660{ }^{\circ} \mathrm{C}$ in an $\mathrm{As}_{2}$ flux to desorb the surface oxide.

${ }^{a)}$ Electronic mail: robert@fys.ku.dk
The Au was then deposited directly onto the surface at $660{ }^{\circ} \mathrm{C}$ and left to anneal for 10 min which resulted in particle sizes of $60-120 \mathrm{~nm}$. The substrate temperature was then lowered to $510{ }^{\circ} \mathrm{C}$ and the GaAs nanowires were grown at a rate of $0.6 \mu \mathrm{m} / \mathrm{h}$ for $20 \mathrm{~min}$ as calibrated by reflection high energy electron diffraction oscillations on a separate calibration piece prior to growth. Finally, the samples were cooled under an As flux. All rods grew in the [111]B direction perpendicular to the surface with a length of approximately $500 \mathrm{~nm}$.

To characterize the nanowires, we performed glancing angle $\mathrm{x}$-ray diffraction with a $(2 \mathrm{D}+2 \mathrm{~S})$ surface diffractometer equipped with a PILATUS2 two-dimensional (2D) (Ref. 11) detector at the Swiss Light Source, PSI. The 2D detector allowed us to sample large three-dimensional (3D) reciprocal space volume data sets which we converted to $\mathrm{I}(h, k, l)$, intensity as a function of coordinates in reciprocal space. We used a set of upstream slits to limit the beam size on the sample but still targeted an ensemble of about $10^{5}$ epitaxial nanowires.

The zinc blende crystal structure of GaAs can be described as an $A B C$ stacking of hexagonal close packed (111) bilayers. Crystal structures consisting of the same (111) bilayers, but with different stacking sequences, have the same Bragg points along the specular (111) direction while the off-specular locations of the Bragg points differ due to the different stacking sequences. To identify different crystal structures along the nanowires we present in Fig. 1 two offspecular scans of intensity in reciprocal space parallel to the [111] wire growth direction. One scan goes through the zinc

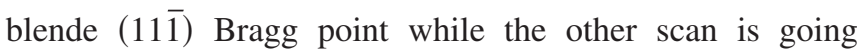
through the (020) Bragg point. These two scans are related by a $60^{\circ}$ rotation around the surface normal, which mirrors the GaAs bulk zinc blende structure $(A B C)$ onto the twinned stacking structure $(B A C)$. Apart from the bulk zinc blende Bragg points at $Q_{\perp}=1((11 \overline{1}))$ and $Q_{\perp}=3((220))$ in one scan and $Q_{\perp}=2((020))$ in the other, the two scans are identical, $Q_{\perp}$ being the momentum transfer in the direction normal to the surface in units of the (111) bulk reflection. In addition to the Bragg points of the regular and twinned stacked zinc blende structures, we also identify Bragg points corresponding to the wurtzite structure and to a six layer stacking re- 


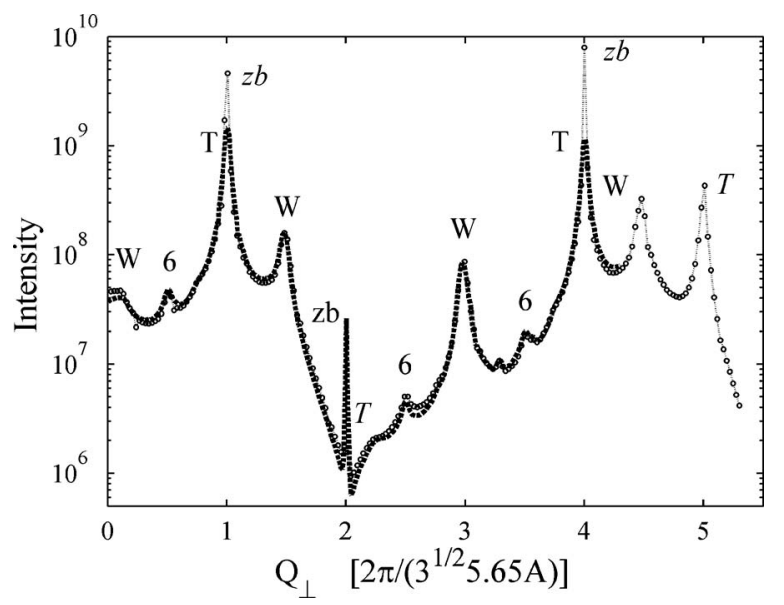

FIG. 1. Two crystal truncation rods parallel to the $[111] B$ growth direction. The different Bragg peaks are labeled. The wurtzite (W) and type 6 (6) Bragg peaks share locations for the two CTRs, while the zinc blende (zb) and twin (T) Bragg peaks are swapped. These are marked with italics for the CTR drawn with open circles/thin dotted line and with regular letters for the CTR drawn as a thick dotted line. The scanning conditions for the two CTRs are identical except for the $60^{\circ}$ rotation of the sample, and the difference in $Q_{\perp}$ range is of no importance.

dundancy (type 6). All of these crystal structures are thus present in the ensemble of nanowires.

The Bragg points are broadened by the small size of the individual crystal structure domains along the wire within each nanowire. From the full width at half maximum $\delta Q$ of the peaks, we can estimate the thickness of each crystal structure along the [111] direction as $L \approx 0.9(2 \pi / \delta Q)$. We find an average thickness of $\sim 160 \AA$ for the twin crystals, $\sim 100 \AA$ for the wurtzite crystals, and a surprisingly large $\sim 150 \AA$ for the type " 6 " crystal, however, part of the intensity for the twin faults is likely to be contributed by "2D" growth on the wafer surface. The regular stacked zinc blende crystal in the nanowires cannot be estimated directly since scattering from the GaAs wafer contributes the majority of the peak intensity to the Bragg point. The tails of the Bragg points, however, mainly arise from the nanowires, and from the symmetry of the zinc blende and twin stacked tails it must be expected that the thickness of the zinc blende layers within the nanowires will be the same as for the twinned stacked parts. From the integrated intensities, we can find the amount of each crystal structure in the nanowires. The ratios of the amounts are derived from the ratio of the integrated intensities while taking Lorentz factors and structure factors into account. ${ }^{12}$ The ratio between the twinned parts relative to the wurtzite structure is 1.6 , and that relative to the 6 structure is 10.6 .

In order to determine the shape and facets of various parts of the nanowires, we exploit the diffuse scattering around the Bragg reflections. A sharp facet will give rise to a diffuse streak of scattering in the direction normal to the facet; such streaks are known as crystal truncation rods ${ }^{13,14}$ (CTRs). By measuring the scattering around the Bragg reflection from each of the crystal structures, we hence get detailed information about the facets of that particular part of the nanowire. In Fig. 2(e) six CTRs emanate from the wurtzite Bragg point at $Q_{\perp}=1.5$. The wurtzite crystal structure is thus bound by six surfaces with $\langle 11 \overline{2}\rangle$ normal direction in terms of the cubic zinc blende basis or $\{10 \overline{1} 0\}$ facets if we refer to the hexagonal wurtzite lattice. In Fig. 2(c), we see a CTR emanate in the [111] direction from the zinc blende Bragg point at $Q_{\perp}=1$. The zinc blende structure is thus bound by $\{111\}$ facets. These are not parallel to the nanowire growth direction and a wire cannot be constructed solely with $\{111\}$ surfaces. The solution is offered by the twin structure (a) which has the same facets as the zinc blende structure but is rotated $60^{\circ}$ around the [111] averaged growth direction. The zinc blende and twin crystal structures thus
$(-101)$ planes

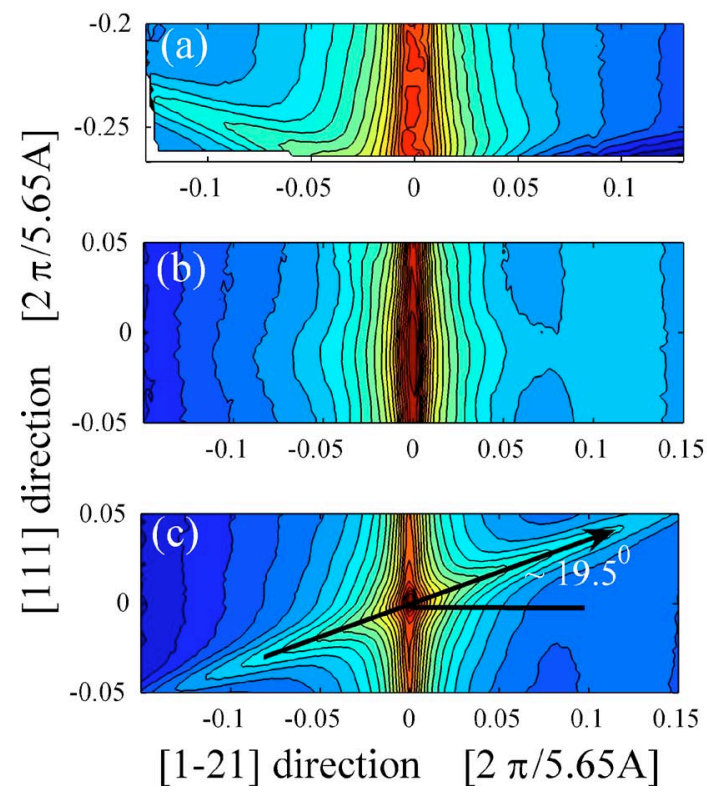

(d)

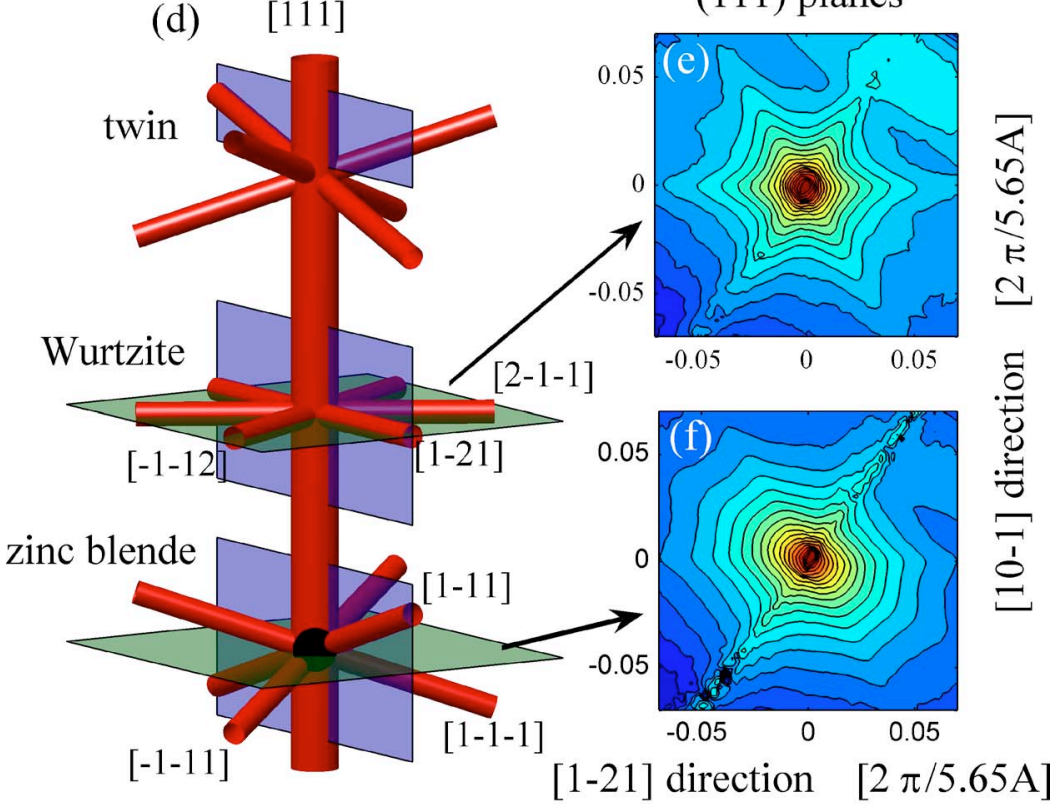

FIG. 2. Reciprocal space maps of Bragg point from [(c) and (f)] the zinc blende, (a) twin, and [(b) and (c)] wurtzite crystal structures show the CTRs from the nanowire facets. (d) The schematic drawing shows how all the CTRs, corresponding to facet surface normals, extend from the different Bragg points. The reciprocal space maps in (a)-(c) correspond to the vertical blue planes in (d), while the [(e) and (f)] reciprocal space maps correspond to the horizontal green planes in (d). 


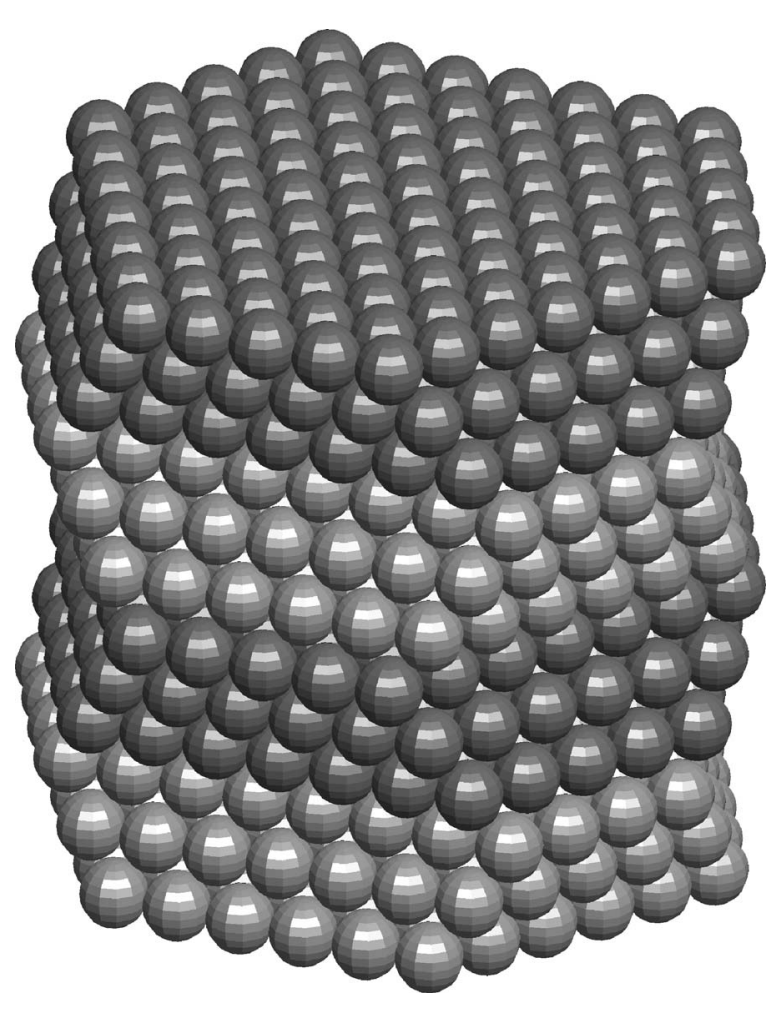

FIG. 3. Ball model of the nanowire as seen from slightly above a $\langle 1 \overline{1} 0\rangle$ direction. The zinc blende (light) and twin (dark) crystals have $\{111\}$ facets and combine to give a microfaceted nanowire. This model was built from a fcc lattice.

combine to form a nanowire with microfacets, as illustrated in Fig. 3, where each slab of zinc blende and twin structures takes the form of a truncated octahedron. Another solution which would fit the crystal truncation rods is the form of a true octahedron, but this does not allow for the hexagonal macrofacets observed in SEM images. The microfaceted structure is the same as that of Johansson et al. ${ }^{10}$ recently found for $\mathrm{GaP}$ nanowires, and for which they proposed a simple thermodynamic model. The model is consistent with our observation that the amount of regular and that of twinned stacked parts of the nanowires are the same.

The composition of the nanowires then serves as an explanation for the twin faults. The $\{111\}$ facets lower the surface free energy but the constraint given by growth at the $\mathrm{Au} /$ nanowire interfaces induces twin faults. In addition, the existence of the type 6 Bragg reflections is explained if the thickness of the twin and zinc blende crystal structures are 3 ML, which results in an $A B C B A C$ stacking, as illustrated in Fig. 3, which is the smallest version possible of a coherent stacking of regular and twinned structures. As seen the amount of type 6 structure is low, but when it occurs it does so in large slabs as the thickness found was surprisingly high. As a consequence, TEM images have not shown clear signatures of this structure but do indicate many faults with a fast repetition sequence. The formation of the wurtzite crystal structure, which is unstable for bulk GaAs, is not explained by the model. We note that the sheer amount of CTRs emerging in precise crystallographic directions excludes the possibilities that the CTRs could arise due to other sources, such as the resolution function, than the facets. X-ray diffraction is ideally suited to study the faulted nanowires since the different crystal structures are fully separated in reciprocal space. X-ray scattering will also be ideal to study the structure and shape of heterostructures.

In conclusion, we have used x-ray diffraction to identify the microfacets of MBE grown GaAs nanowires as a combination of $\{111\}$ surfaces from zinc blende and the twin structure, with the macrofacets giving a hexagonal cross section bound by $\{11 \overline{2}\}$ facets. In small parts of the wire, the twin and zinc blende structures combine to produce a $6 \mathrm{ML}$ thick superlattice. In agreement with the microfaceted model symmetry arguments indicate similar thickness of zinc blende and twin structures within the wires. In addition the nanowires contain a large amount of the hexagonal wurtzite structure bound by $\{10 \overline{1} 0\}$ facets, and we have found that the amount of twin structure is around 1.6 times the amount of wurtzite structure.

The authors acknowledge discussion with Erik Johnson and Poul Erik Lindelof. We also thank Christian Schlepuetz for his technical assistance. The work is supported from the Danish Natural Science Research Council.

${ }^{1}$ P. Yang, Y. Wu, and R. Fan, Int. J. Nanosci. 1, 1 (2002).

${ }^{2}$ L. J. Lauhon, M. S. Gudiksen, and C. M. Lieber, Philos. Trans. R. Soc. London, Ser. A 362, 1247 (2004).

${ }^{3}$ L. Samuelson, C. Thelander, M. T. Björk, M. Borgström, K. Deppert, K. A. Dick, A. E. Hansen, T. Mårtenson, N. Panev, A. I. Persson, W. Seifert, N. Sköld, M. W. Larsson, and L. R. Wallenberg, Physica E (Amsterdam) 25, 313 (2004).

${ }^{4}$ B. A. Wacaser, K. Deppert, L. S. Karlsson, L. Samuelson, and W. Seifert, J. Cryst. Growth 287, 504 (2006).

${ }^{5}$ M. C. Plante and R. R. LaPierre, J. Cryst. Growth 286, 394 (2006).

${ }^{6}$ A. I. Persson, M. W. Larsson, S. Stenströmm, B. J. Ohlsson, L. Samuelson, and R. Wallenberg, Nat. Mater. 3, 677 (2004).

${ }^{7}$ J. C. Harmand, G. Patriarche, N. Péré-Laperne, M.-N. Mérat-Combes, L. Travers, and F. Glas, Appl. Phys. Lett. 87, 203101 (2005).

${ }^{8}$ A. Mikkelsen, N. Sköld, L. Ouattara, M. Borgstöm, J. N. Andersen, L. Samuelson, W. Seifert, and E. Lundgren, Nat. Mater. 3, 519 (2004).

${ }^{9}$ Z. Zhu, T. Andelman, M. Yin, T.-L. Chen, S. P. O'Brien, and R. M. Osgood, J. Mater. Res. 20, 1033 (2005).

${ }^{10}$ J. Johansson, L. S. Karlsson, C. P. T. Svensson, T. Mårtensson, B. A. Wacaser, K. Deppert, L. Samuelson, and W. Seifert, Nat. Mater. 5, 574 (2006).

${ }^{11}$ E. F. Eikenberry, Ch. Brönniman, G. Hülsen, H. Toyokawa, R. Horisberger, B. Schmitt, C. Schulze-Briese, and T. Tomizaki, Nucl. Instrum. Methods Phys. Res. A 501, 260 (2003).

${ }^{12}$ E. Vlieg, J. Appl. Crystallogr. 30, 532 (1997).

${ }^{13}$ I. K. Robinson, Phys. Rev. B 33, 3830 (1986).

${ }^{14}$ M. Nielsen, D. Smilgies, R. Feidenhans'l, E. Landemark, G. Falkenberg, L. Lottermoser, L. Seehofer, and R. L. Johnson, Surf. Sci. 352/354, 430 (1996). 